TI 2003-018/3

Tinbergen Institute Discussion Paper

Risk Aversion and the Subjective Time Discount Rate:

A Joint Approach

Bernard M.S. van Praag

Adam S. Booij

Faculty of Economics and Econometrics, University of Amsterdam, and Tinbergen Institute. 


\section{Tinbergen Institute}

The Tinbergen Institute is the institute for economic research of the Erasmus Universiteit Rotterdam, Universiteit van Amsterdam, and Vrije Universiteit Amsterdam.

Tinbergen Institute Amsterdam

Roetersstraat 31

1018 WB Amsterdam

The Netherlands

Tel.: $\quad+31(0) 205513500$

Fax: $\quad+31(0) 205513555$

Tinbergen Institute Rotterdam

Burg. Oudlaan 50

3062 PA Rotterdam

The Netherlands

Tel.: $\quad+31(0) 104088900$

Fax: $\quad+31(0) 104089031$

Please send questions and/or remarks of nonscientific nature to driessen@tinbergen.nl.

Most TI discussion papers can be downloaded at http://www.tinbergen.nl. 


\title{
RISK AVERSION AND THE SUBJECTIVE TIME DISCOUNT RATE: A JOINT APPROACH*
}

\author{
BERNARD M.S. VAN PRAAG ${ }^{\dagger}$ AND ADAM S. BOOIJ
}

\section{Tinbergen Institute and Department of Economics and Econometrics, University of Amsterdam}

In this paper we analyze a large sample of individual responses to six lottery questions. We derive a simultaneous estimate of risk aversion $\gamma$ and the time preference discount rate $\rho$ per individual. This can be done because the consumption of a large prize is smoothed over a larger time period. It is found that $\rho$ and $\gamma$ strongly vary over individuals, while they are negatively correlated with a correlation coefficient of -.3 . Furthermore we explain $\gamma$ and $\rho$ by income, age, gender, entrepreneurship and an obesity index. Very significant effects are found. If we explain $\gamma$ in a simple model where time discounting is ignored, we find completely different estimates for $\gamma$. We conclude that in the case of lotteries with big prizes a simultaneous estimate of $\gamma$ and $\rho$ is needed in order to avoid misspecification.

\footnotetext{
${ }^{*}$ We thank Joop Hartog and Peter Wakker for their helpful comments on an earlier version.

${ }^{\dagger}$ Corresponding author: University of Amsterdam, Roeterstraat 11, 1018 WB Amsterdam, The Netherlands; Email:B.M.S.vanPraag@uva.nl, Tel: (0031) 20525 6018, Fax (0031) 205256013.
} 


\section{Introduction}

Choice behavior of economic agents is hampered in practice by two complicating phenomena. First, the outcome of our decisions may be a random variable. Examples are investment under uncertainty, buying insurance policies or shares and participation in lotteries. Second, many decisions have consequences for the future. They do not only affect present utility but future utility as well. Examples are again investments, the purchase of durable goods and savings.

For both problems there are standard solutions. Decisions under uncertainty are described by the Von Neumann - Morgenstern (VNM) model, which defines the utility to be maximized as the expectation of the utilities of the random alternatives. The VNM - model is not beyond discussion. We refer to Allais (1953), Kahneman and Tversky (1979), Tversky and Kahneman (1992) and Wakker and Tversky (1993) for critique and alternatives. Within the VNM approach an important ingredient is the specification of the utility function. The most popular one-parameter specification is the Constant Relative Risk Aversion (CRRA) function where the (cardinal) utility function is characterized by the risk aversion parameter $\gamma$. Empirical estimates for $\gamma$ vary from 1.5 to 8 . Most studies of risk aversion look either at gambles (see e.g. Jullien and Salanié (2000), Beetsma and Schotman (2001), Hartog, Ferrer- i - Carbonell and Jonker (2002)) or at decisions on the choice of risky assets in portfolios or the choice of insurance policies (Halek and Eisenhauer (2001), Kapteyn and Teppa (2002), Donkers, Melenberg and Van Soest (2001)). Subjective measures of risk aversion obtained through hypothetical questions have also been used to explain choice under uncertainty (see, for example, Barsky, Juster, Kimball and Shapiro (1997), Kapteyn and Teppa (2002)).

A very similar model, the discounted utility model, describes the problem of decisions over time where utilities at different moments of time are weighted by a subjective time discount rate $\rho$. This model is also not beyond discussion as is clear from the critique of Loewenstein and Prelec (1992). The not so many empirical estimates of the parameter $\rho$ vary a great deal. They are mostly derived from consumption - smoothing models (Trostel and Taylor (2001)), experimental choice situations (e.g. Read (2001), Benzion, Rapoport, Yagil(1989)) or hypothetical questions (see e.g. Lazaro, Barberan and Rubio (2001)). 
It is rather evident that many of these problems are in fact ridden by the two complications simultaneously. Decisions reach over time and they are risky. Obstfeld (1994) observes that 'unless risk aversion and intertemporal substitutability are carefully separated, attempts to measure the welfare cost of changes in consumption risk can yield misleading conclusions about the role of risk aversion. Under expected-utility preferences, which make no such separation, an increase in risk aversion simultaneously alters the effective discount factor ....'.

It follows that such situations have to be described by a utility function $\sum_{s, t} p_{s, t} u_{s, t}(\rho, \gamma)$, where we sum over the random states $s$ and the moments of time $t$. The subject of this paper will be the joint estimation of the time discount rate and relative risk aversion. According to our knowledge there are only a few and scattered publications with respect to this simultaneous approach and a small number of estimates. We refer to Abdulkadri and Langemeier (2000) who try to estimate both coefficients simultaneously using farm consumption data obtaining estimates of the elasticity of intertemporal substitution between 0.158 and 0.351 and estimates of relative risk aversion between 2.849 and 6.329. Another example is the joint estimation of relative risk aversion, the discount rate and the elasticity of substitution by Issler and Piqueira (2000) using macro-consumption data of the Brazilian economy. Their results with respect to the discount rate seem robust at between .8 and .9 per year whereas their estimates of relative risk aversion are very sensitive to the model specification used.

There are various empirical frameworks where we actually attempt to estimate such models. The first source is observation of real life situations, where we observe households, firms or traders on the stock exchange. It is well known that this is a notorious problem in practice. The quality of the data is frequently weak and the number of observations not very large and/or selective. Another source of data is experiments in experimental economics laboratories. The evident advantage of the experimental setting is that we can formulate the decision problem and vary it at will, while observing the respondent's reactions to changes. In practice such experiments have a lottery structure. A drawback with this way of data collection is the artificial environment, the relatively small numbers of observations that can be realized, and the fact that in practice the respondents are mostly students who cannot be seen as representative for the population at large. Finally, as the prizes are mostly paid out to make things more realistic, necessarily the prizes will be small money. However, it may well be possible that behavior 
differs with the seriousness of the price. Especially for small amounts the tendency will be to consume the price within a few days without any saving. For large prizes this will be different and we may assume an effect of time discounting. However, the problem with real experiments is that large prizes are too costly to pay out in practice.

Hence, we see that all the situations described have their drawbacks. Either there are a small number of observations, or the empirical description and observation is problematic or there are problems with the relevance of the observations for the test persons are in laboratory settings. Sometimes, a variety of the objections holds at the same time. This is not intended as a critique, since the ideal situation is difficult to find in reality. However, it points to the fact that we have to compromise. A final point that may be surmised is whether it is reasonable to assume that the risk aversion used in small lotteries with a slight chance on a prize should have to be the same as the risk aversion on which we base major investment decisions. Is there a possibility that the underlying behavioral model used is context-dependent?

Necessarily the present paper also starts from a compromise. In this paper we use a large anonymous survey in which we ask for the bidding price for six different lotteries (cf. Hartog, Ferrer-i-Carbonell, Jonkers (2001) who use the same data source). Given the fact that we have six lotteries, it is possible to simultaneously identify an approximate value for the respondent's relative risk aversion $\gamma$ and the respondent's subjective discount rate $\rho$. It turns out that there is a correlation of about -0.35 between the two values. Given the fact that our sample consists of about 8,000 respondents, we can explain the variations in $\gamma$ and $\rho$ by some individual characteristics like income, age, education, gender, intensity of religious participation, entrepreneurship, the individual's Quetelet index and other variables. Most effects are very significant and plausible. In terms of variance explained the results are poor. It is evident that this data collection mode has his shortcomings as well. We may be sure that most respondents did not spend much time in answering the questions, as these were fictitious lotteries without any real possible rewards or losses. Nevertheless, we can assume that nearly all respondents have been co-operative and that they have not reported nonsensical answers. We did, however, drop those respondents who were responding in a strange or irrational way, for instance, when they would pay a higher price than the expected value. 
The structure of this paper is as follows. In the next section we describe the data, followed by the description of the behavioral model in Section 3. In Section 4 we present and analyze the survey results. In Section 5 we conclude with a discussion and an evaluation. 


\section{The data.}

Our data have been collected in a somewhat unorthodox way. In the Netherlands nearly all daily journals are distributed on a subscription basis, where readers have a strong link with their journal. There are about six national dailies, which are focusing on national and international news; they are identifiable as belonging to the right or the left of the political spectrum. Next to those six journals there are a score of regional dailies, which are widely distributed on a subscription basis among the households of a specific city or region. A sizeable minority is subscriber to a 'national' and a 'regional' at the same time. The 'regionals' focus on local news, but they cover national and international news as well. With a view on the cost of an international network of correspondents the regional dailies share the international network. Therefore they are member of a combination, the Joint Press Services (GPD) at The Hague. Once in about four years since 1983 the GPD- dailies print an extensive questionnaire ${ }^{1}$ in their Saturday issue, consisting of about thousand items. The readership, consisting of over 2 million households, is invited to fill out the anonymous questionnaire, and to return it to their journal by mail, unstamped. We notice that this procedure differs in several aspects from usual procedures. First, the questionnaire is rather long, second no person gets a reminder when it is not returned, and third the respondent does not get a gift for responding. In short, the predictions for response must be bleak. Indeed the response is about $2 \%$, which is comparable to the response on direct mail campaigns. Although this percentage seems low, the absolute number of respondents is huge. There are about 40,000 persons who return the questionnaire within a fortnight with genuine enthusiasm. The results are presented and analyzed in the regional journals. Mostly the survey is done shortly before general elections, such that the results can help citizens in making their voting decision and can inform the politicians about the opinions among the population.

Of course we may doubt whether such a survey is representative for the Dutch population. The first obvious restriction is that respondents have to be subscribed to a journal. It was found that the readership is a good reflection of the Dutch population minus the ethnic minorities who are not subscribing and in many cases do not speak and write Dutch sufficiently and minus the truly poor who do not read a regularly journal. The sample of respondents are somewhat older

\footnotetext{
${ }^{1}$ The GPD-surveys have been conducted in 1983, 1984, 1991, 1998 and 2001. They have been designed and analyzed by B.M.S. van Praag and varying co -authors, starting with the late Aldi Hagenaars.
} 
and more educated than the average, but after the usual re-weighting the sample may be considered as representative for the Dutch population minus the two segments mentioned. The GPD-samples have been used for government-commissioned studies and have been the basis for several scientific research projects. For the study in this paper we do not need and, consequently, are not interested in the representativity issue.

The questionnaire may be seen as a dragnet. It does not focus on one issue but it has to contain various issues, which fascinate various segments of the population in order to get as many different respondents as possible. The expectation is that somebody, who likes to give his opinion about a specific item, will fill out the whole questionnaire. So there is a module on the living situation of the household, on who cares for the children, a module about the job of the respondent and that of his or her partner, a module about money spending, about time-spending, about the living environment, health and a host of questions inviting respondents to evaluate politicians and various aspects of politics.

The question module on which we are concentrating for this paper is that of the 'lottery questions' (Saturday January 17, 1998, q.104). This module runs as follows.

Suppose that a lottery ticket is offered to you for a lottery in which ten people participate (so you have a chance of 1 in 10 that you will win). The prize is a money amount equal to your wage per month. What is the maximum amount ${ }^{l}$ you are willing to pay for the ticket? Dfl......

Please also answer this question for the following lotteries:

\begin{tabular}{|ll|}
\hline Five people participate, prize: DFl.1,000 & I pay Dfl...... \\
\hline Five people participate, prize: one monthly wage & I pay Dfl....... \\
\hline Ten people participate, prize: Dfl. 5,000 & I pay Dfl....... \\
\hline Hundred people participate, prize: Dfl. 1,000 & I pay Dfl...... \\
\hline Hundred people participate, prize: Dfl.1 million & I pay Dfl...... \\
\hline
\end{tabular}

\footnotetext{
${ }^{1}$ Notice that this is in Dutch guilders as the EURO was introduced afterwards on January 1, 2002. A EURO is about one USA \$, while a EURO is 2.2 Dutch guilders.
} 
The six lotteries differ with respect to the chances $\pi$ which equal $1 / 5,1 / 10$ and 1/100 and with respect to the size of the prize $Z$. Four prices are in absolute amounts ranging from DFl. 1000 to Dfl. 1.000.000. Unlike most lotteries in experimental contexts, where prizes vary between $\$ 10$.and \$200.- the prizes in this case are not small money. The million guilder prize particularly large and will be equal to between 10 and 15 years income for most respondents. The other two prizes vary with the respondent as they are in fact stated in terms of the respondent's monthly wage. As we have an estimate of monthly income for each respondent this is no problem for our analysis. It is obvious that this formulation yields a greater variability in the prize range. 


\section{The Model.}

Let us assume that an individual has a constant monthly net income of $y$, and that he gets an offer to participate in a lottery, which will give a prize $Z$ with a chance $\pi$. Moreover let the price of a ticket be denoted by $\tilde{A}$ and his utility function be denoted by $U(\cdot)$. If individual preferences satisfy the Von Neumann-Morgenstern (NM) axioms, then the expected utility of accepting that offer will be

$$
(1-\pi) U(y-\tilde{A})+\pi U(y-\tilde{A}+Z) .
$$

The maximum amount an individual is prepared to pay for taking part in the lottery is that amount $A$, which solves the equation

$$
(1-\pi) U(y-A)+\pi U(y-A+Z)=U(y) .
$$

We call $A$ the value of the lottery or the reservation price. The problem becomes more complex if we assume a multi-period model. We assume that the individual has an income flow $y(t)$ and that the price of the ticket has to be paid in the period $[0,1]$ but that the prize, if won, is consumed in parts $p(t) Z$ over the period $(1, \infty)$ where

$$
\int_{1}^{\infty} p(t) d t=1 \text { and } p(t) \geq 0 \text { for all } t
$$

The time unit is taken to be a month, as some of the prizes are expressed in monthly wage ${ }^{1}$. In that case the value of the lottery is found by solving the equation

$$
\int_{0}^{1} e^{-\rho t} U(y-A) d t+\pi e^{-\rho} \int_{1}^{\infty} e^{-\rho t} U(y+p(t) Z) d t+(1-\pi) \int_{1}^{\infty} e^{-\rho t} U(y) d t=\int_{0}^{\infty} e^{-\rho t} U(y) d t,
$$

\footnotetext{
${ }^{1}$ This is an arbitrary assumption. If we take another time unit like two weeks or two months, we do not find very different results. For $\rho$, which depends on the time unit, we have to make a simple correction. We thought that a month would conform rather well with the payout time expected by the respondent and with the actual payout time of Dutch lotteries.
} 
where $\rho$ stands for the subjective time discount factor. Moreover, we assume in the context of buying a lottery ticket that the respondent assumes that his income $y$ and his utility function $U(\cdot)$ remain constant over life ${ }^{1}$.

It follows that the value of $A$ also depends on the way in which the prize is spent over time. If we assume that the consumption pattern for the windfall gain can be spent at will, it follows that the value of the lottery is found from the equation

$$
\frac{\left(1-e^{-\rho}\right)}{\rho}(U(y)-U(y-A))=\pi \max _{p(\cdot)} \int_{1}^{\infty} e^{-\rho t} U(y+p(t) Z) d t-\int_{1}^{\infty} e^{-\rho t} U(y) d t .
$$

The answer $A$ that is given by the respondent to the lottery question can thus be seen as the respondent's solution to the above equation (5). As we have six lottery questions we find for each respondent $n$ six solutions $A_{i n}$ where $i=1, \ldots, 6$. If we want to derive information from them with respect to personal parameters with respect to time preference and risk aversion, we shall have to make some assumptions, which make our model empirically tractable.

The value of monthly net household income will be denoted by $y_{n}$ or $y$, if no confusion can arise. Moreover we assume that utility functions will be of the Constant Relative Risk Aversion type (CRRA) as introduced by Arrow (1965) and Pratt (1964). That is, $U(y)=y^{(1-\gamma)} /(1-\gamma)$ where $\gamma>0$. If $\gamma=1$, then this function is defined as $U(y)=\ln (y)$.

We may now find the optimum pattern of how the prize $Z$ should be spent over the future periods. Using the continuous formulation (4), the Euler-conditions and the constraints in (3) imply the optimal spending pattern,

$p(t)=\left\{\begin{array}{ccl}C e^{B t}-\psi & \text { if } & 1 \leq t \leq T_{\max } \\ 0 & \text { if } & t>T_{\max }\end{array}\right.$

\footnotetext{
${ }^{1}$ This seems a reasonable hypothesis for most readers, who fill in a large questionnaire on their Saturday evening and who do not use sophisticated models on their future income flow.
} 
with $B \equiv-\frac{\rho}{\gamma}<0, \psi \equiv \frac{y}{Z}>0$ and two remaining parameters, $C$ and $T_{\max }$. Notice that this implies that $y+p(t) Z=y+\left(C e^{B t}-\frac{y}{Z}\right) Z=C e^{B t} Z$. It follows that the maximum itself is the integral

$$
\begin{aligned}
& \int_{1}^{T_{\max }} e^{-\rho t} \frac{\left(C e^{B t} Z\right)^{1-\gamma}}{(1-\gamma)} d t=\frac{(C Z)^{1-\gamma}}{(1-\gamma)} \int_{1}^{T_{\max }} e^{-\rho t+B t(1-\gamma)} d t \\
& =\frac{(C Z)^{1-\gamma}}{(1-\gamma)} \int_{1}^{T_{\max }} e^{B t} d t=\frac{(C Z)^{1-\gamma}}{(1-\gamma)} \frac{1}{B}\left(e^{B T_{\max }}-e^{B}\right),
\end{aligned}
$$

where we used the definitions of $B$ and $\psi$ to simplify the expression.

As $p(t)=C e^{B t}-\psi$ is monotonically decreasing in $t$, we find that the spending share would eventually become negative. This implies that the time length $T_{\max }$ during which the prize is spent is finite. We have $p\left(T_{\max }\right)=0$. As the shares integrate (or sum) to one, we may solve for the two parameters $C$ and $T_{\max }$. As we see from equation (6) the maximum spending time $T_{\max }$ depends on $B \equiv-\frac{\rho}{\gamma}<0$ and the 'relative prize' $\psi \equiv \frac{y}{Z}>0$. In order to get some idea of how $T_{\max }$ varies with the two parameters we present Table 1 . We see that $T_{\max }$ increases with the relative prize. For instance, let us assume an individual with a monthly income of 2000 with $\gamma$ equal to 2 and a time discount of $2 \%$ (per month). The spending period of a prize of hundred times his monthly income, that is 200.000 will then be 115 months, that is, nearly 10 years. Notice that for most configurations the spending period will be fairly short. The prize is then considered as a windfall profit, to be consumed almost immediately. We also observe that it is the ratio of $\gamma$ to $\rho$ which is important and not the absolute values of those parameters. 
Table 1: $T_{\max }$ for different preferences and different relative prizes.

\begin{tabular}{c|l|lllll}
\hline \multicolumn{2}{c}{} & \multicolumn{5}{c}{$\psi$} \\
\cline { 3 - 7 } \multicolumn{2}{c}{} & 0,01 & 0,1 & 1 & 10 & 100 \\
\hline \multirow{3}{*}{$B \quad$} & -100 & 1,09 & 1,07 & 1,05 & 1,03 & 1,01 \\
& -10 & 1,69 & 1,47 & 1,26 & 1,11 & 1,04 \\
& -1 & 5,66 & 3,61 & 2,15 & 1,42 & 1,14 \\
& $-0,1$ & 27,11 & 12,46 & 5,16 & 2,38 & 1,44 \\
& $-0,01$ & 115,62 & 42,62 & 14,82 & 5,44 & 2,41 \\
\hline
\end{tabular}

In a discrete-time context we would find a solution 'between two discrete moments in time', which would imply a rounding-off procedure. It is therefore that we prefer a continuous model. Consequently we can evaluate the maximum in equation (5) and solve equation (4) for $A$ given $\rho$ and $\gamma$, where we assume that the lottery ticket has to be paid uniformly over the first period and that the outcome of the (hypothetical) lottery is known and the payment of the prize will be realized after one month. More precisely we have

$$
A_{\text {in }}=A\left(\gamma_{n}, \rho_{n} ; y_{n}, \pi_{i}, Z_{\text {in }}\right) \quad(i=1, \ldots, 6)
$$

We are now interested in estimating $\gamma$ and $\rho$ for each respondent $n$. This will be done in the next section.

\section{The estimates.}

We may consider (8) as a system of six non-linear equations in the two unknowns $\gamma_{n}$ and $\rho_{n}$ where the $A_{i n}$ stand for the predicted six responses to the six questions. We notice that $Z_{1 n}$ and $Z_{3 n}$ depend on $n$, since the prizes in lotteries 1 and 3 are formulated in terms of $y_{n}$. It is obvious that we cannot find an exact solution $(\hat{\gamma}, \hat{\rho})$ for system (8) as we have only two unknowns and six equations to be satisfied. Hence we minimized the sum 


$$
S_{n}^{u}\left(\gamma_{n}, \rho_{n}\right)=a_{n}^{\prime} a_{n}
$$

with respect to $\gamma_{n}$ and $\rho_{n}$, where $a_{n}^{\prime}=\left(a_{1 n}, \ldots, a_{6 n}\right)$ is the error vector with elements $a_{i n}=\ln \mathrm{A}_{i n}-\ln A\left(\gamma_{n}, \rho_{n} ; y_{n}, \pi_{i}, Z_{i n}\right)$ and $\mathrm{A}_{\text {in }}$ corresponds to the observed reservation price of lottery $i$ for individual $n$. This yields 'unweighted average' values $\left\{\gamma_{n}^{u}, \rho_{n}^{u}\right\}_{n=1}^{N}$, where we ignored the rare responses where the ticket prize exceeded the lottery expectation. In those cases the average was calculated on less than six responses. We found that responses were moderately correlated. Hence we calculated the average covariance matrix. We denote the 6-vector of the unweighted 'residuals' by $a_{n}^{u}$ and we define the average residual covariance-matrix $\Sigma_{A}^{u}$

$$
\Sigma_{A}^{u}=\frac{1}{N} \sum_{n=1}^{N} \Sigma_{A, n}^{u}=\frac{1}{N} \sum_{n=1}^{N} \frac{1}{6} a_{n}^{u} a_{n}^{u \prime}
$$

The corresponding variance/correlation matrix, where we present the variances on the diagonals and the correlation coefficients on the off-diagonal entries, is presented in Table 2.

Table 2: The unweighted covariance/correlation matrix of the response residuals

\begin{tabular}{l|rrrrrr}
\hline & \multicolumn{1}{|l}{$a_{1}^{u}$} & $a_{2}^{u}$ & $a_{3}^{u}$ & $a_{4}^{u}$ & $a_{5}^{u}$ & $a_{6}^{u}$ \\
\hline$a_{1}^{u}$ & .32 & & & & & \\
$a_{2}^{u}$ & -.30 & .28 & & & & \\
$a_{3}^{u}$ & .22 & .02 & .27 & & & \\
$a_{4}^{u}$ & -.07 & .03 & .24 & .24 & & \\
$a_{5}^{u}$ & -.39 & -.34 & -.63 & -.49 & .71 & \\
$a_{6}^{u}$ & -.46 & -.00 & -.61 & -.45 & .47 & .05 \\
\hline
\end{tabular}

It is seen that the correlation between some residuals is considerable. Therefore we recalculated the individual's $\gamma_{n}$ and $\rho_{n}$ by minimizing the weighted sum of residuals

with respect to $\left(\gamma_{n}, \rho_{n}\right)$.

$$
S_{n}^{w}\left(\gamma_{n}, \rho_{n}\right)=a_{n}^{\prime}\left[\Sigma_{A}^{u}\right]^{-1} a_{n}
$$


In this way we get our revised individual estimates $\left\{\gamma_{n}^{w}, \rho_{n}^{w}\right\}_{n=1}^{N}$ where the superscript indicates the weighting. Obviously this is a rather crude method as we estimate the two individual parameters $\gamma_{n}$ and $\rho_{n}$ from six observations, which are presumably error-ridden and where the errors are likely to be correlated. It is obvious that large-sample theory does not apply and hence we did not bother to construct standard deviations of the individual parameters. However as our number of observations is rather large, $N=9420$, we may assume that the sample as such contains reliable information.

The re- weighted residuals $a_{i n}^{w}$ have a covariance matrix, which appeared practically equal to that of the unweighted residuals. First we shall look at some primary statistics. The sample averages and standard deviations are given in Table 3 and in Figures 1.a and 1.b we depict the histograms of $\gamma$ and $\rho$.

Table 3. Mean and standard deviation of $\gamma$ and $\rho$.

\begin{tabular}{|c|c|c|c|c|c|}
\hline Variable & $\mathrm{N}$ & average & Stand.dev. & minimum & maximum \\
\hline$\gamma$ & 9420 & 1.54 & 3.78 & 0.01 & 39.99 \\
\hline$\rho$ & 9420 & 2.41 & 1.41 & 0.01 & 6.99 \\
\hline
\end{tabular}

\section{Figure 1. Histogram of Estimated variables.}

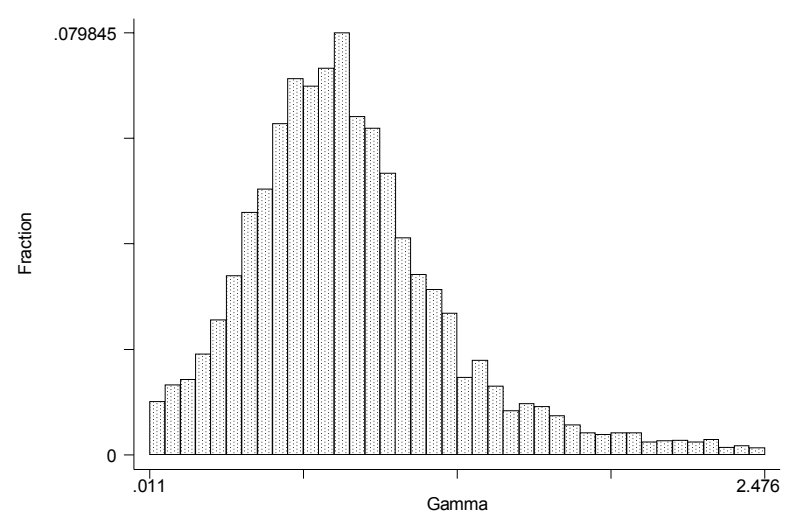

(a) Histogram of $\gamma$.

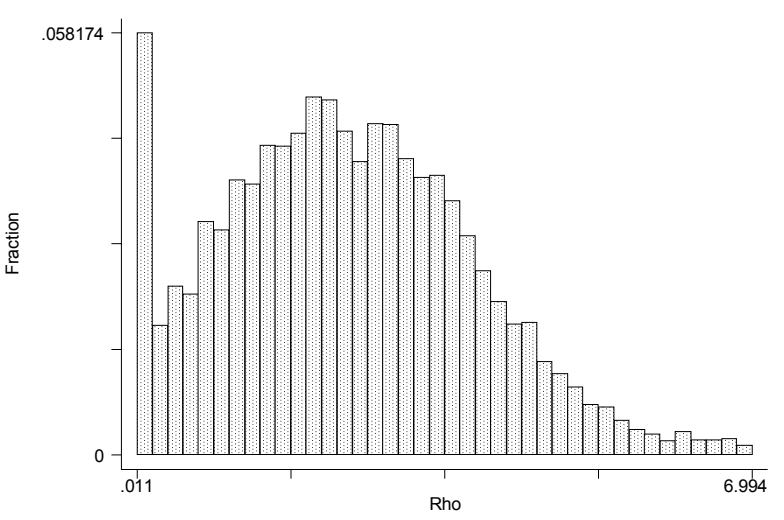

(b) Histogram of $\rho$. 
We see that the distribution of $\gamma$ is rather skew and looks, if anything, log- normal. The distribution of $\rho$ is also skew. There is a sizeable part of $\rho$ estimates that are virtually equal to zero. There is also a fat right-tail. The latter class of respondents does not take into account that there is no need to consume the lottery prize immediately, but that consumption may be smoothed over a longer period of time. As a matter of fact five of the offered lotteries have a prize, which will be of the same order of magnitude as a monthly wage. This does not invite to serious savings for the future. It follows that it might be that the subjective time discount rate used is context-dependent. For major investments we take into account that the harvest may be reaped over a long period and that consumption may be gradual over time. For relatively small 'windfall profits' many individuals will not bother about saving but consume it immediately. For those individuals the time discount will be very high. If we normalize the time discount, which implies a weighting density $\frac{1}{\rho} e^{-\rho t}$, we can calculate the median value of the distribution for $\rho=2.41$ at 0.56 . It implies that the median lies at about two weeks. This gives an idea about the time horizon, which is used by most respondents in this context. This finding makes it doubtful whether individuals use the same time discount in every context. For decisions, which have heavy and long-term implications like, for instance, the choice of one's marriage partner, we may assume that $\rho$ will be much smaller. We shall not try to investigate this conjecture within the scope of this paper.

This being said, let us look at the correlation between $\gamma$ and $\rho$. We find a moderate negative correlation coefficient of -.34 . This indicates firstly that the two concepts are clearly separately identifiable, but secondly that high-risk aversion goes hand in hand with low time discounting. This is precisely what we expect of prudent people. They take few risks and they look a long time ahead. 


\section{Explanations.}

In this section we shall make an attempt to explain the values found by some independent variables. (See for other examples Pålsson (1996), Halek \& Eisenhauer (2001), Hartog, Ferrer-iCarbonell and Jonker (2002)). We try to explain $\ln (\gamma)$ and $\ln (\rho)$ by a SUR-model of the type

$$
\begin{aligned}
& \ln (\gamma)=\beta_{\gamma}^{\prime} x+\varepsilon_{\gamma} \\
& \ln (\rho)=\beta_{\rho}^{\prime} x+\varepsilon_{\rho}
\end{aligned}
$$

As our knowledge about these relationships is almost nil, we shall not attempt to formulate sophisticated theories on why we use these linear relationships. We take recourse to the literature and to our own intuition. Moreover we are of course dependent on the information in our sample.

First, we introduce (monthly) income $y$ as a variable measured in Dutch guilders. Our idea is that risk aversion will decline with increasing income as the same monetary risk becomes relatively less important when income increases. For the same reason we may expect that individuals with a lot of income will use a higher time discount rate to evaluate the windfall profit of the lottery prize.

The second variable is age measured in years. On one hand older people are more settled and hence can take more risks. On the other hand older people are more cautious and take less risks. With respect to the time discount we observe that with aging the remaining lifetime shortens. This would suggest a shrinking time horizon and hence a stronger time discounting.

A third variable is education, measured as the number of years spent on regular education. First, we have to realize that education is human capital. It is a powerful instrument to cope with the risks of life. Hence, individuals who do not see the risks of life are less interested in education as a means to neutralize those risks. Second, individuals with more education have more information and hence are more risk-aware. For both reasons we assume that more educated people are more risk-averse. Similarly, education is a long-term investment and such a long-term investment is triggered by a long time horizon. Hence, we assume that more education goes hand in hand with a lower time discount. 
Males (a dummy equal to 1) are assumed to be less risk-averse than females (dummy equals zero). We do not have any idea about the gender effect on time discounting.

Religion is also a way to cope with the risks of life. Accepting the religious rules gives a feeling of safety and security. Hence, individuals with greater risk aversion will tend to be more religious. Given the orientation on the hereafter in some religions we might expect also an effect on time discounting. We included a measure of religiousness, which varies over five categories, where 1 stands for non-religious and 5 for very religious.

The essence of the entrepreneur is that he accepts more risks than the ordinary human being. Hence, we expect a lower risk aversion for entrepreneurs.

Obvious variables are whether the respondent is working or has no paid activity. If he has not, we assume that he is more 'living by the day' implying a smaller time horizon, i.e., larger $\rho$.

For someone living single we assume the same, as his life may be less planned than respondents living with a family.

Although we assume that the number of children is related to time discounting, we do not have an idea how. It may be that individuals give less weight to the future or that they give more. The same holds for children and risk aversion. Clearly, getting children is a risky choice. We would expect that families with children are less risk averse. On the other hand, if you like to conform to society and you would not like to be lonesome, a good solution is to get children. Such people are typically risk averse.

Finally, we know the length and the weight of the respondents, from which we may infer a measure of obesity, the so-called Quetelet-index. It is defined as the ratio of the weight in $\mathrm{kg}$, divided by the square of the length in meters. This is the generally accepted obesity index. Here again it is difficult to predict a relationship between risk aversion and obesity. It is evident that eating too much has risky consequences. However, eating too much may also be explained by a precautionary motive, because one is afraid to become hungry; that is risk aversion. For the 
relation between obesity and time preference we can think of obesity as a manifestation of 'saving in kind'. Then it would follow that individuals with a low time discount rate would 'save' much.

Now it is time to let the data speak. In Table 4 we present the regression estimates of both equations. The observed $\gamma$ and $\rho$ appear to be negatively correlated with a correlation ratio of -.34 . Hence, we apply Seemingly Unrelated Regression.

We see that most coefficients are statistically significant. Indeed age and income have the predicted effects. Although we include log-income and its square in the explanation of $\ln (\rho)$ the top of the parabola is found for an astronomically high income. Hence time preference is increasing in $y$. The difference in risk aversion between males and females is non-significant contrary to what is found in the literature. The gender effect on time discounting is very strong. Males are much more future looking than females.

Table 4: Regression estimates of $\ln (\gamma)$ and $\ln (\rho)$ (SUR- regression).

\begin{tabular}{lccc|llll}
\hline $\ln (\gamma)$ & Coef. Std. Err. & t-stat. & $\ln (\boldsymbol{\rho})$ & Coef. Std. Err. & t-stat. \\
\hline $\ln (y)$ & -0.052 & 0.021 & -2.560 & $\ln (y)$ & 1.893 & 0.393 & 4.810 \\
$\ln ($ Age $)$ & -0.228 & 0.032 & -7.040 & $\ln ^{2}(y)$ & -0.083 & 0.018 & -4.510 \\
$\ln (E d u)$ & 0.189 & 0.047 & 3.980 & $\ln (A g e)$ & 0.297 & 0.047 & 6.260 \\
Male & -0.038 & 0.021 & -1.790 & $\ln (E d u)$ & -0.306 & 0.059 & -5.190 \\
RELLevel & 0.024 & 0.009 & 2.720 & Male & -0.303 & 0.026 & -11.540 \\
Entrepeneur & -0.150 & 0.040 & -3.770 & Work & -0.136 & 0.025 & -5.370 \\
$\ln (Q)$ & 0.141 & 0.071 & 2.000 & $\ln (\mathrm{Q})$ & -0.206 & 0.087 & -2.370 \\
Constant & 0.358 & 0.337 & 1.060 & $\ln (\#$ Child $)$ & 0.034 & 0.019 & 1.790 \\
& & & & Single & -0.158 & 0.028 & -5.570 \\
& & & Constant & -9.613 & 2.138 & -4.500 \\
\hline$N=8504$ & $R^{2}=0.01$ & & $N=8504$ & $R^{2}=0.04$ & \\
\hline
\end{tabular}

The effects of religions are as expected. Religious people are more risk averse and more aware of the future. Workers appear to have a lower time discount rate than non-actives as we argued 
above, the number of children on the other hand does not have a significant effect on risk aversion contrary to what we anticipated.

Being single has a very marked influence on the time discount rate, which we did not expect. Singles have a wider time horizon than respondents living with a partner or within a household.

Finally, the obesity index has very marked effects both on $\gamma$ and on $\rho$. Obesity is strongly correlated with a higher risk aversion and a smaller time discount. Indeed, eating much may be interpreted as 'saving in kind'.

Although these regression results are significant and interesting we must confess that the $R^{2}$ of the first equation is 0.01 and that of the second equation is 0.04 . Hence the explanatory value of these models is not overwhelming. However, it is well-known that nearly all estimates, based on cross - section micro-data, are plagued by the same phenomenon. Indeed, there must be a lot of unobserved heterogeneity. Moreover, the type of questions that we employ are and cannot be very accurately responded. It is stated behavior. The respondent is a Saturday evening-reader of a daily, who likes to fill out the rather complex questionnaire, but who can hardly be expected to devote much thinking to each separate question. What we register are 'first sentiments'. The advantage is that the respondent does not strive for social desirability, etc. The disadvantage is that there will be a considerable amount of white noise in the answers.

The correlation between the two errors $\varepsilon_{\gamma}$ and $\varepsilon_{\rho}$ is estimated to be -.60 . Hence, it seemed wise to use the SUR-option. Nevertheless, we feel that the results cannot be discarded as being non-significant or intuitively implausible.

In the analysis of risk aversion $\gamma$ is mostly explained in isolation (from now denoted by $\gamma^{s}$ ), that is, without taking into account the time aspect of decisions. Therefore we performed the same analysis on the simple model where the individual is expected to behave as if he was solving the standard lottery model, given in equation (12)

$$
\pi U(y-A+z)+(1-\pi) U(y-A)=U(y)
$$


Some Descriptive statistics are given in Table 5 and the resulting regression equation is given in Table 6.

Table 5: Mean and standard deviation of $\gamma^{s}$.

\begin{tabular}{|c|c|c|c|c|c|c|}
\hline Variable & $\mathrm{N}$ & average & Stand.dev. & minimum & Median & maximum \\
\hline$\gamma^{s}$ & 9365 & 41.88 & 71.29 & .005 & 20.52 & 1677.79 \\
\hline
\end{tabular}

Table 6: Regression, based on the standard Von Neumann-Morgenstern model.

\begin{tabular}{llrr}
\hline $\ln \left(\gamma^{s}\right)$ & Coef. Std. Err. & t-stat. \\
\hline $\ln (y)$ & 0.602 & 0.029 & 20.980 \\
$\ln ($ Age $)$ & 0.587 & 0.045 & 12.940 \\
$\ln ($ Edu $)$ & -0.469 & 0.066 & -7.100 \\
Male & -0.399 & 0.030 & -13.470 \\
RELLevel & 0.051 & 0.015 & 3.270 \\
Entrepreneur & -0.240 & 0.069 & -3.480 \\
$\ln (\mathrm{Q})$ & -0.082 & 0.099 & -0.830 \\
Constant & -4.110 & 0.471 & -8.720 \\
\hline$N=8792$ & $R^{2}=0.0765$ & \\
\hline
\end{tabular}

Figure 2: Histogram of Estimated $\gamma^{s}$.

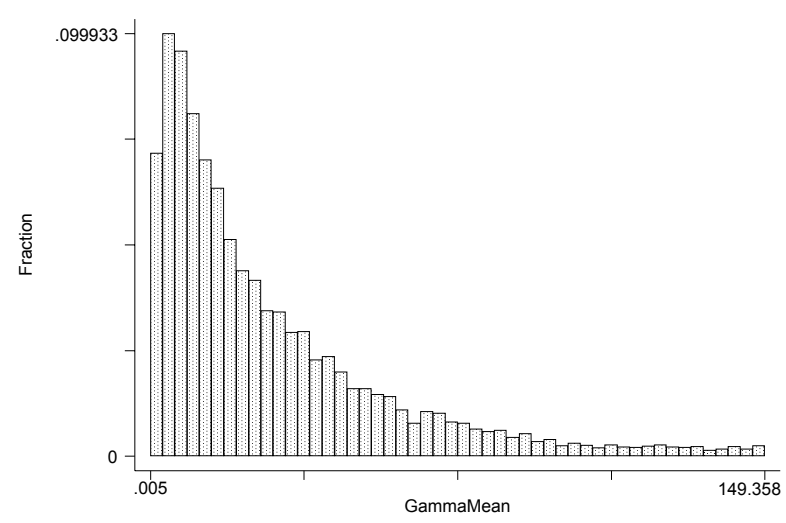

We see that nearly all coefficients appear to be highly significant, except the obesity index. However, we find coefficients that are completely different from those we found in Table 4 for the $\gamma$-equation. For instance, the negative age effect of -.228 is reversed to a positive.587. A similar change is found for the education effect. In both specifications we find a negative entrepreneurial effect. Our conclusion is that the latter specification must be seen as a serious misspecification, which yields completely different effects. Of course, we do not try to implicate that our two-equation model is the correct specification, but we derive from these results that the one-equation model is certainly much worse.

Finally, we considered the correlation between the two $\ln (\gamma)$-estimates per individual. We found a negative correlation between both estimates of -0.29 . The correlation between the two 
$\gamma$ 's (without the logs) is only -0.09 . Hence, it seems that both estimates of $\gamma$ measure different things. 


\section{Conclusion}

In this paper we looked at various lottery questions that, separately from the risk dimension, have a time dimension. That is, the evaluation of the prize does not only depend on the absolute amount of the prize but also on the way in which the prize may be gradually spent over time. It is intuitively obvious that this plays a greater role for big prizes $Z$ than for small ones. For instance, somebody with an income of $\$ 2000$,- per month will consider a prize of $\$ 10$,- as 'small' but he will consider a prize of one million dollars as large. For large prizes it is probable that the consumption will be smoothed in order to optimize the consumption of the prize over time. In our view it is unavoidable to conclude that in general risk aversion cannot be separately estimated from lottery questions. So risk aversion should always be estimated jointly with the subjective time discount rate except for very small prizes. However, what is 'small' depends on the personal circumstances of the respondent.

This finding generalizes to many other settings, where we think of risky assets, portfolios, etc. Obviously, this also holds inversely. If we try to estimate subjective time discount rates from the evaluation of risky assets over time, we cannot do this without simultaneously taking the attitude towards risk into account.

The second conclusion, which we may draw, is that our subjective time discount estimates are very high, at least compared to what is generally assumed in the literature. Although it is now recognized that the subjective time discount rate and the market interest rate are two different things and that the subjective concept is much higher, the average we find would imply a time half - value of about two weeks which is obviously a very short time horizon. Our explanation is that the time discount rate is case - and context - dependent. First, if the decision to be taken or the choice to be made has no long-term consequences, individuals will reduce their time horizon. For instance, if the prize is so small, that it will be consumed with certainty in one year from now, the individual will not bother with the period farther away and truncate the time weight distribution. The time discount distribution utilized is case-dependent. The weighting system, which is used, is not the true time discount distribution but a truncated version of it. We surmise that the horizon is shorter as the ratio $(Z / y)$ is smaller. 
Next to that there is presumably a context - dependence. If the individual has to make a serious decision this requires an effort of the mind. One such an effort is to realize what your time preference distribution is (approximately), because you are not daily thinking of it. It is a second effort to look for an optimal spending program. Hence, if we look at hypothetical survey questions where nothing really is at stake, we may not suppose that respondents make a very serious effort in answering the question. And again, a truncation of the time horizon is an efficient way to reduce the response effort.

Hence, we assume that the same method will yield more plausible results for the time discount rate if we propose lotteries, which require a wider time horizon. This may be generated by, formulating prizes, which are not an incidental windfall profit, but which affect the individuals income flow over lifetime. For instance a doubling of income over the remaining lifetime. (see Barsky et al.).

Summarizing, we believe that this is a fruitful approach, but that it can still be improved in a number of directions. 


\section{References}

Abdulkadri, A.O., Langemeier, M.R. (2000). 'Using farm consumption data to estimate the Intertemporal Elasticity of Substitution and Relative Risk Aversion coefficients.', Agricultural Finance Review 60, p.61-70

Allais, M. (1953). 'Le Comportement de l'Homme Rationnel devant le Risque, Critique des Postulats et Axiomes de l'École Américaine', Econometrica 21: p.503-46.

Arrow, K.J. (1965). Aspects of the Theory of Risk Bearing, Helsinki: Yrjö Jahnssonin Saato

Barsky, R., Juster, F.T., Kimball, M.S. and Shapiro, M.D. (1997), 'Preference Parameters and Behavioral Heterogeneity. An Experimental approach in the Health and Retirement Study.', The Quarterly Journal of Economics 112, p.537-80.

Beetsma, R.M.W.J., and P. C. Schotman, (2001). 'Measuring risk Attitudes in a Natural Experiment: Data from the Television Game Show Lingo', The Economic Journal 111, p.821- 848 .

Benzion, U., Rapoport, A., Yagil, J., (2002). 'Discount Rates Inferred from decisions: An Experimental Study', Management Science 35, p.270-84.

Donkers, B., Melenberg B. and Van Soest, A. (2001) 'Estimating Risk Attitudes using Lotteries: A Large Sample Approach', The Journal of Risk and Uncertainty 22, p.165-95.

G.P.D. Survey 1998. Created by H. Maassen van de Brink, B.M.S. van Praag and Geassocieerde Pers Diensten, The Hague.

Halek M, Eisenhauer, J.G. (2001), 'Demography of risk Aversion', Journal of Risk and Insurance, 68, p.1-34.

Hartog J., Ferrer- i - Carbonell, A. and Jonker, N. (2002). 'Linking Measured risk Aversion to Individual characteristics', Kyklos, 55, p.3-26.

Issler, J.,V. and Piqueira, N.S., (2000), 'Estimation Relative Risk Aversion, the Discount Rate, and the Intertemporal Elasticity of Substitution in Consumption for Brazil Using Three Types of Utility Function', Brazillian Review of Econometrics 20, p.201-39.

Jullien, B. and Salanié, B. (2000), 'Extimating Preferences under risk: The case of Racetrack Bettors', Journal of Political Economy 108, p.503-29.

Kahneman, D. and Tversky, A. (1979). 'Prospect Theory: An Analysis of Decision under Risk', Econometrica 47, p.263-92. 
Kapteyn, A. and Teppa, F. (2002), 'Subjective Measures of risk Aversion and Portfolio Choice', working paper, CentER, Tilburg.

Lazaro, A., Barberan, R., Rubio, E. (2001). 'Private and Social Time Preferences for Health and Money: An empirical Estimation', Health Economics Letters 10, 351-56.

Loewenstein, G. and Prelec D. (1992). 'Anomalies in Intertemporal Choice: Evidence and an Interpretation', The Quarterly Journal of Economics 107, p.573.97.

Obstfeld, M. (1994), 'Evaluating risky consumption paths: The role of intertemporal substitutability', European Economic Review, 38, p.1471-86

Pålsson, A. (1996). 'Does the degree of relative risk aversion vary with houshold characteristics?', Journal of Economic Psychology 17, p.771-87

Pratt, J.W., (1964), 'Risk aversion in the small and in the large', Econometrica, 32, p.122-136

Read, D. (2001), 'Is Time - Discounting Hyperbolic or Subadditive?', Journal of risk and uncertainty, 23, p.5-32.

Trostel, P.A. and Taylor, G.A. (2001), 'A theory of Time Preference', Economic Inquiry 39, p.379-95.

Wakker, P. and Tversky, A. (1993). 'An axiomatization of cumulative prospect theory', Journal of risk and uncertainty 7, p.147-76. 